\title{
Memoria de Trabajo y Planificación en niños: validación de la prueba Torre de Londres
}

\author{
Mémoire de travail et planification chez l'enfant : validation de la tâche de la Tour de Londres \\ Memória de trabalho e planejamento em crianças: validação da tarefa Torre de Londres \\ Working Memory and Planning in children: Validation of the Tower of London Task
}

Irene Injoque-Ricle ${ }^{1} \&$ Débora I. Burin ${ }^{1}$

${ }^{1}$ Consejo Nacional de Investigaciones Científicas y Técnicas (CONICET)

Universidad de Buenos Aires, Argentina.

\begin{abstract}
Resumen
Memoria de trabajo (MT) y planificación son dos aspectos de la Función Ejecutiva que están íntimamente relacionados. El objetivo de este trabajo fue explorar la asociación entre los distintos componentes del modelo de Baddeley y Hitch de MT y la planificación en niños, y observar si esta asociación varía con la edad. Para ello se realizaron dos estudios. El Estudio 1 tuvo como objetivo validar de forma definitiva una versión local de la prueba Torre de Londres (TOL). Se administraron TOL y distintas medidas externas a 210 niños de 6, 8 y 11 años de la Ciudad Autónoma de Buenos Aires, Argentina. Se comprobó la validez y confiabilidad de la versión local de TOL, haciendo que la prueba sea apta para ser utilizadas con niños de dichas edades de la Ciudad Autónoma de Buenos Aires, Argentina. En el Estudio 2 se llevó a cabo el objetivo principal del trabajo. Se administró TOL y la Batería Automatizada de Memoria de Trabajo (AWMA) a 180 niños de 6, 8 y 11 años. Se encontraron asociaciones significativas entre las puntuaciones de TOL y las puntuaciones de la AWMA que representan al Ejecutivo Central de la MT en los niños de 8 años, y entre TOL y las puntuaciones del Ejecutivo Central y de la Agenda Viso-Espacial de la AWMA en los niños de 11 años. Los datos permiten concluir que el componente verbal de la MT no estaría implicado en la resolución de la tarea TOL, y que si bien el componente viso-espacial está involucrado en niños grandes, sería el componente ejecutivo el que más peso tiene para la resolución de esta tarea de planificación. Palabras-claves: Memoria de trabajo; AWMA; planificación; Torre de Londres; niños.
\end{abstract}

\section{Résumé}

La mémoire de travail (MdT) et la planification sont deux aspects des fonctions exécutives et sont intimement reliées. Le but de cette recherche était d'explorer les liens entre les différentes composantes du modèle de la MdT de Baddeley et Hitch et la planification chez l'enfant et d'explorer si ces associations variaient selon son âge. Deux études ont été réalisées. L'étude 1 avait pour but de valider une version locale de la tâche de la Tour de Londres (TOL). 210 enfants de la ville de Buenos Aires en Argentine, âgés de 6, 8 et 11 ans ont été évalués à cette tâche et à différents tests. De bons coefficients de validité et de fidélité ont été mis en évidence à la TOL, faisant de cette tâche, un instrument valide pour l'évaluation des enfants âgés de 6, 8 et 11 ans de la ville de Buenos Aires en Argentine. L'étude 2 s'intéresse plus directement à l'objectif principal de cette recherche. 180 enfants de la ville de Buenos Aires en Argentine, âgés de 6, 8 et 11 ans ont été évalués à la TOL et la Batterie Automatisée d’Evaluation de la Mémoire de Travail (AWMA). Des corrélations significatives ont été retrouvées entre les scores évaluant l'administrateur central de la MdT à la TOL et à l'AWMA pour un sous-groupe d'enfants de 8 ans. De plus, d'autres corrélations significatives ont été retrouvées entre les scores évaluant l'administrateur central et le calepin visuo-spatial de la MdT à la TOL et à la AWMA pour le groupe d'enfants de 11 ans. Ces résultats permettent de conclure que la composante verbale de la MdT ne serait pas impliquée dans la résolution de la tâche de la TOL. De plus, même si le calepin visuo-spatial est impliqué dans la résolution de cette tâche chez les enfants plus âgés, l'administrateur central de la MdT reste la composante nécessaire pour résoudre cette tâche de planification.

Mots-clefs : Mémoire de travail ; AWMA ; planification ; Tour de Londres ; enfant

Nota de los autores:

Esta investigación fue financiada por el Consejo Nacional de Investigaciones Científicas y Técnicas (CONICET; Res. № 258/06 y Res. N ${ }^{\circ}$ 3100/08) y por la Secretaría de Ciencia y Técnica de la Universidad de Buenos Aires (UBACyT P016). La Batería Automatizada de Memoria de Trabajo (AWMA) fue traducida y adaptada bajo permiso. Copyright @ 2007 de Harcourt Assessment, Copyright @ 2007 de la traducción al español de Harcourt Assessment. Todos los derechos reservados. The Automated Working Memory Assessment battery was translated and reproduced by Permission. Copyright (c) 2007 by Harcourt Assessment. Spanish Translation copyright @ 2007 by Harcourt Assessment. All rights reserved.

Artículo recibido: 25/04/2011; Artículo revisado: 19/07/2011; Artículo aceptado: 30/08/2011.

Irene Injoque-Ricle, CONICET - Facultad de Psicología, Universidad de Buenos Aires, Argentina. Débora I. Burin, CONICET - Facultad de Psicología, Universidad de Buenos Aires, Argentina.

Correspondencia relacionada con este artículo debe ser enviada a: Irene Injoque-Ricle, Instituto de Investigaciones, Facultad de Psicología, Universidad de Buenos Aires. Av. Independencia 3056, $3^{\circ}$ piso (C1425AAM), Ciudad Autónoma de Buenos Aires, Argentina. Tel.: +54-11-49575886.

E-mail: iinjoque@psi.uba.ar

DOI: $10.5579 / \mathrm{rnl} .2011 .0065$ 
Memória de trabalho (MT) e planejamento são dois aspectos das funções executivas e são muito relacionadas. O objetivo deste estudo foi explorar a relação entre diferentes components do modelo de MT de Baddeley e Hitch e planejamento em crianças, além de investigar se esta associação varia entre diferentes faixas etárias na infância. Dois estudos foram realizados. O Estudo 1 visou a validar uma versão local da tarefa Torre de Londres (TL). TL e diferentes tarefas externas foram administradas em 210 crianças de 6, 8 e 11 anos de idade de Buenos Aires, Argentina. Bons coeficientes de validade e de fidedignidade desta versão local da TL foram obtidos, demonstrando que esta tarefa é válida para avaliação de crianças de 6, 8 e 11 anos de idade, provenientes de Buenos Aires, Argentina. No Estudo 2, o principal objetivo deste artigo foi explorado. TL e a Automated Working Memory Assessment Battery (AWMA) foram utilizados para o exame de 180 crianças de 6, 8 e 11 anos de idade. Associações significativas entre os escores da TL e da AWMA que representam o executivo central da MT foram encontradas no subgrupo de 8 anos de idade, e entre os escores da TL e os escores da AWMA que representam o executivo central e a alça visuoespacial da MT foram observadas no grupo de 11 anos de idade. Tais achados sugerem que o component verbal da MT não estaria envolvido na resolução da tarefa TL, e mesmo que a alça visuoespacial esteja envolvida em crianças maiores, o executivo central é o componente com maior destaque na resolução desta tarefa de planejamento.

Keywords: Memória de trabalho; AWMA; planejamento; Torre de Londres; crianças.

Función Ejecutiva (FE) es un constructo teórico multidimensional. Lezak (1995) lo define como las habilidades que permiten realizar de manera eficaz conductas con un propósito, dirigidas a una meta. Otros investigadores como Shallice (1990) o Stuss (1992) concuerdan con este planteo. Si bien todavía no hay un consenso en qué habilidades cognitivas se agruparían en las funciones ejecutivas, muchos autores coinciden en que están compuestas por tres componentes separados, pero integrados: control atencional -atención selectiva y sostenida-; flexibilidad cognitiva -memoria de trabajo, cambio atencional y automonitoreo-; y establecimiento de metas -iniciación, planificación, resolución de problemas y conducta estratégica(Bull, Espy, \& Senn, 2004; Lezak, 1995; Luria, 1973; Miyake, et al., 2000; Neisser, 1967; Shallice, 1990; Soprano, 2003; Stuss, 1992; Walsh, 1978). Estas habilidades son importantes para un funcionamiento cotidiano eficaz, y alteraciones de esta función provocarían problemas atencionales, de planificación, en la implementación de estrategias adecuadas y de flexibilidad del pensamiento (Anderson, Anderson, Northam, Jacobs, \& Mikiewicz, 2002). Las regiones neuroanatómicas que sustentan la FE se localizan en la corteza prefrontal (Boghi, et al., 2006; Newman, Carpenter, Varma, \& Just, 2003; Stuss \& Alexander, 2007). Evidencia fisiológica muestra que existe un proceso de mielinización de las fibras nerviosas, y de maduración de las estructuras frontales durante la niñez y la adolescencia (Fuster, 2002). Asociado a este proceso de maduración, los niños y adolescentes pueden ir adquiriendo gradualmente la capacidad para realizar procesamientos de manera más eficiente, debido a que la transmisión de la información nerviosa se vuelve más rápida con el aumento de mielina en los axones (Fuster, 2002). El desarrollo incompleto de los lóbulos frontales durante la infancia y la adolescencia puede implicar una habilidad limitada para realizar procesos ejecutivos.

La Memoria de Trabajo (MT) es un sistema de memoria activo responsable del almacenamiento temporal y procesamiento simultáneo de información necesaria para la realización de tareas cognitivas complejas (Baddeley, 1986; Baddeley, Eysenck, \& Anderson, 2009). Permite comprender y representar mentalmente el entorno inmediato, retener información sobre sus experiencias pasadas inmediatas, apoyar la adquisición de conocimiento, resolver problemas yformular, relacionar y actuar en base a metas actuales (Baddeley \& Logie, 1999). El modelo más influyente de MT es el desarrollado por Baddeley y Hitch (Baddeley, 1986, 2007; Baddeley \& Hitch, 1974), en el que se plantea que en el centro se encuentra el Ejecutivo Central, amodal y sin capacidad de almacenamiento, que controla y regula los dos subsistemas esclavos: la agenda viso-espacial, que almacena material visual y espacial, y el bucle fonológico, que almacena información basada en el lenguaje. Los tres subcomponentes del modelo de MT han sido relacionados con una gran variedad de procesos cognitivos complejos, tanto en la adultez como en la infancia. Algunos de estos procesos son la adquisición del lenguaje, de nuevo vocabulario, la comprensión lectora, la adquisición de habilidades aritméticas, la realización de cálculos mentales, el aprendizaje de rutas espaciales y la planificación (Alloway, Gathercole, Willis, \& Adams, 2004; Anderson, et al., 2002; Ashcraft, 1995; Burin, Duarte, Prieto, \& Delgado, 2004; Cohen, 1996; Gathercole, Alloway, Willis, \& Adams, 2006; Swanson \& Jerman, 2007). En el año 2000, Baddeley actualizó el modelo, e incorporó un tercer subsistema, el Buffer Episódico, que sirve de interface entre diferentes sistemas, cada uno de los cuales involucran distintos tipos de códigos (Baddeley, 2000).

Otro aspecto clave de la FE es la planificación, que implica la capacidad de los sujetos de generar y organizar la secuencia de pasos necesarios para realizar una tarea a partir de una meta propuesta (Lezak, 1995; Soprano, 2003). Esta habilidad está íntimamente relacionada con la inhibición, la flexibilidad cognitiva y la MT, ya que para poder planificar de manera eficaz es necesario analizar alternativas posibles, elegir la adecuada e inhibir las otras, y mantener en la memoria el plan generado (Bull, et al., 2004). Las pruebas que evalúan la planificación suelen requerir tareas en las que hay que llegar a una meta a partir de reglas que constriñen el espacio de movimientos o pasos, pero sin un camino predeterminado, y que deben realizarse en un tiempo límite (Bull, Espy \& Wiebe, 2008; Lezak, 1995; Shallice, 1990). Las pruebas más empleadas para evaluar planificación son laberintos y los tests de torres -como la de Hanoi o de Londres- (Bull, et al., 2008; Lezak, 1995).

Como se dijo antes, las FE se desarrollan desde la temprana infancia. Si bien el desempeño en pruebas de FE aumenta con la edad, las habilidades y procesos que se incluyen en este constructo tienen tiempos evolutivos diferentes. Esto explicaría la variabilidad en el desempeño de los sujetos de una misma edad entre las distintas pruebas que evalúan la FE (Bull, et al., 2004; Bull, et al., 2008; Soprano, 2003), y pone de manifiesto la necesidad de contar con pruebas específicas para cada aspecto de la FE, adecuadas para cada período del desarrollo. En la prueba de Torre de Londres (TOL, Shallice, 1982), se encontró que la eficiencia en el desempeño aumenta con la edad, desde los tres años de edad hasta los doce o catorce años, donde se equipara al desempeño adulto (Krikorian, Bartok, \& Gay, 1994b; Lipina, Martelli, Vuelta, Injoque Ricle, \& Colombo, 2004; Steinberg, 2007). Se ha propuesto que el desarrollo de la planificación estaría ligado al de la MT (Cohen, 1996; Hayes-Roth \& Hayes-Roth, 1979; Owen, Downes, Sahakian, Polkey, \& 
Robbins, 1990). Cohen (1996) plantea que la MT es importante en la formulación, retención e implementación de los planes, y también en su revisión on-line. Específicamente, en la prueba TOL, es plausible que el armado, la mantención y la ejecución de un plan que implica múltiples etapas, implique una alta demanda de recursos de la MT (Phillips, Wynn, Gilhooly, Della Sala, \& Logie, 1999). Philips y cols. (1999) plantearon que si bien el formato de presentación y de respuesta de TOL es viso-espacial, no es claro si el proceso de planificación implica cargas en los recursos de MT verbal y/o viso-espacial. Muchos autores han destacado el rol de la MT viso-espacial en la resolución de TOL (e.g. Joyce \& Robins, 1991; Morice \& Delahunty, 1996; Owen, Doyon, Petrides, \& Evans, 1996; Temple, Carney, \& Mullarkey, 1996; Welsh, Cicerello, Cuneo, \& Brennan, 1995). Welsh y cols. (1995) realizaron entrevistas a sujetos adultos luego de haber sido evaluados con la Torre de Hanoi -prueba en la cual se basa TOL-. Los participantes reportaron que visualizaban mentalmente los movimientos de los discos en las varillas. Un argumento que contrasta con estos hallazgos es el de Morris, Ahmed, Syed y Toone (1993), quienes sostienen que existe un repaso verbal activo involucrado en la realización de TOL. Estudios de activación cerebral realizados durante el desempeño de la tarea TOL mostraron una activación significativa del lóbulo frontal izquierdo (Morris, et al., 1993; Owen, et al., 1996). Si bien es una simplificación relacionar la activación del hemisferio izquierdo exclusivamente con el lenguaje, Morris y cols. sostuvieron a partir de estos hallazgos que el grado de lateralización encontrado durante el desempeño en TOL sugería un papel de la memoria de trabajo verbal en la resolución de la tarea.

Así pues, la MT participa en la resolución de pruebas de planificación como TOL. Sin embargo, cabe preguntarse acerca de la participación de la memoria de trabajo verbal y viso-espacial, que supondrían respectivamente verbalizar (de manera abierta o silente) el plan de acción, o visualizar una secuencia de movimientos. El objetivo de este trabajo ha sido explorar la asociación entre los distintos componentes de la MT y la planificación en niños, y ver si esta asociación variaba con la edad. Para ello, en primer lugar, se realizó una validación definitiva de una versión de TOL preliminarmente validada (Injoque-Ricle \& Burin, 2008b). Los resultados de este trabajo se presentan en el Estudio 1, mientras que los resultados que responden al objetivo principal del artículo se exponen en el Estudio 2.

\section{Estudio 1}

\section{Método}

\section{Participantes}

Se trabajó con 210 sujetos de ambos sexos (122 mujeres $-58.1 \%$ - y 88 varones), de clase media, distribuidos en tres grupos de 70 niños cada uno, uno de 6 , otro de 8 y otro de 11 años de edad (ver Tabla 1). Estos niños asistían a dos escuelas primarias públicas de la Ciudad Autónoma de Buenos Aires, Argentina (99 escuela A -47.1\%- y 111 escuela B). Fueron excluidos de la muestra niños repitentes, con problemas de aprendizaje, trastornos neuropsicológicos, psiquiátricos, auditivos o del lenguaje y aquellos con un CI igual o inferior a 69.

Revista Neuropsicologia Latinoamericana (2011), 3(2), 31-38
El acceso a las instituciones se realizó con el aval de los directivos de las mismas, y los niños participaron luego de haber obtenido la autorización escrita de los padres, tras una reunión informativa en la que se les explicó los objetivos de la investigación, la metodología de trabajo, y se les aseguró la confidencialidad de la información y el anonimato de los participantes.

Tabla 1

Distribución por sexo según grupo de edad de la muestra de adaptación

\begin{tabular}{lcccccc}
\hline & \multicolumn{7}{c}{ Edad } \\
\cline { 2 - 7 } & \multicolumn{2}{c}{6} & \multicolumn{2}{c}{8} & \multicolumn{2}{c}{11} \\
\cline { 2 - 7 } & $\mathrm{N}$ & $\%$ & $\mathrm{n}$ & $\%$ & $\mathrm{~N}$ & $\%$ \\
\hline Mujeres & 33 & 47.1 & 39 & 55.7 & 50 & 71.4 \\
Varones & 37 & 52.9 & 31 & 44.3 & 20 & 28.6 \\
\hline Total & 70 & 100 & 70 & 100 & 70 & 100 \\
\hline
\end{tabular}

\section{Instrumentos}

Torre de Londres (TOL; Injoque-Ricle \& Burin, 2008b; Shallice, 1982). Es una tarea de planificación y resolución de problemas que para ser resuelta de manera eficaz implica la puesta en marcha de procesos como organización de la tarea, iniciar el plan y sostenerlo en la memoria durante su realización, inhibir posibles distractores y cambiar de estrategia de modo flexible en aquellos casos que sea necesario. Es una modificación realizada por Shallice (1982) a partir de la prueba Torre de Hanoi (Klahr, 1978; Simon, 1975). Se les presenta a los sujetos dos aparatos con tres esferas de distintos colores -rojo, azul y amarillo- y tres varillas de diferentes tamaños cada uno -una grande en la que entran tres esferas, una mediana en la que entran dos, y una pequeña en la que sólo entra una-. A partir de una configuración inicial -por ejemplo, la esfera azul en la varilla pequeña, la amarilla en grande, y la roja encima de esta última-, deben alcanzar una configuración final determinada por ejemplo, la esfera amarilla en la vara pequeña, la azul en la mediana y la roja en la grande-, siguiendo las reglas presentadas por el examinador: que sólo pueden mover una esfera por vez, y que por lo tanto, nunca pueden tener más de una esfera en la mano al mismo tiempo, que tienen que hacerlo en la cantidad de movimientos indicados -en nuestro ejemplo, 4-, y en el menor tiempo posible. Para lograr un buen desempeño en TOL, un sujeto debe tener habilidades de planificación eficientes para resolver el problema en la menor cantidad de movimientos posibles.

El material utilizado consistió en dos aparatos de madera iguales, con una base de base $26 \mathrm{~cm}$. de largo x 4.5 $\mathrm{cm}$. de ancho y $3.5 \mathrm{~cm}$. de alto, con tres varillas de $0.8 \mathrm{~cm}$. de diámetro y 4.5, 9 y $15 \mathrm{~cm}$. de largo, respectivamente; cada uno con tres esferas de madera de $5 \mathrm{~cm}$. de diámetro, de colores rojo, amarillo y azul.

Para el diseño de la versión local de TOL se realizó una adaptación preliminar de la tarea (ver Injoque-Ricle \& Burin, 2008b). En dicha adaptación se administró primero un pool inicial de 48 ensayos, divididos en ocho niveles. A medida que aumentaban los niveles crecía en uno la cantidad de movimientos necesarios para resolver cada ensayo. Esta versión fue administrada sin criterio de corte para poder realizar un análisis de los ítems. Para ello se midió su dificultad. Se crearon originalmente seis ensayos por nivel 
para poder seleccionar dentro de cada nivel los tres ítems con similar nivel de dificultad y poder obtener finalmente una tarea con niveles de dificultad creciente. El nivel ocho tuvo que ser eliminado ya que sus ensayos presentaban la misma dificultad que los ensayos del nivel cuatro.

La versión definitiva de la prueba, administrada en este estudio, está compuesta por siete niveles de dificultad creciente, con tres ensayos cada uno. Para pasar al nivel siguiente, que implica problemas con un movimiento más que el anterior, el sujeto tiene que haber resuelto de manera eficaz al menos dos de los tres ensayos. Un ensayo se considera correcto cuando se alcanzó la configuración final con la cantidad mínima de movimientos requeridos y dentro del tiempo máximo estipulado para cada nivel. Se otorga un puntaje ponderado para cada ensayo correcto en función del nivel en el que se encuentra. Por ejemplo, cada ensayo correcto del nivel 1 otorga un punto, cada ensayo correcto del nivel 2 otorga dos puntos, y así sucesivamente. Luego se suman las puntuaciones parciales obteniendo así un puntaje total de la prueba.

Batería Automatizada de Memoria de Trabajo (AWMA; Alloway, 2007; Injoque-Ricle, Calero, Alloway, \& Burin, 2011). Es un instrumento compuesto por 12 pruebas que miden los diferentes aspectos de la MT y se administran utilizando una computadora. Las pruebas que evalúan la capacidad de almacenamiento de información verbal de la MT son Amplitud de dígitos, Amplitud de palabras y Amplitud de no-palabras. Las tareas de la AWMA que permiten medir la capacidad de almacenamiento de información espacial son Matrices dinámicas y Amplitud viso-espacial. La capacidad de almacenamiento de información visual es evaluada con Laberintos. Para medir la capacidad de procesamiento de información verbal se utilizan Amplitud de oraciones, Amplitud numérica y Amplitud de dígitos inverso. Por último, las pruebas que evalúan la capacidad de procesamiento de información espacial son Figura diferente, Señor $X$ y Recuerdo espacial. Cada prueba cuenta con niveles de dificultad creciente, entre los cuales va aumentando de uno en uno la cantidad de estímulos por ensayo. Cada nivel tiene seis ensayos, y para pasar de nivel el sujeto tiene que haber hecho adecuadamente al menos cuatro ensayos de un mismo nivel. La descripción detallada de las pruebas se encuentra en Injoque-Ricle y cols. (2011). Con respecto a las propiedades psicométricas de la batería, todas las pruebas cuentan con buenos índices de fiabilidad (Alpha de Cronbach entre .81 y .95) y con evidencias a favor de validez concurrente y discriminante (ver Injoque-Ricle, et al., 2011).

Serie de Dibujos (SD; Injoque-Ricle \& Burin, 2008a). Su objetivo es evaluar el reconocimiento visual de dibujos a través de la capacidad de almacenamiento de información visual en la MT. Se le presenta al niño una serie de dibujos familiares en una hoja, que pueden variar de tres a nueve, según el nivel en el que se encuentre. Luego se presenta una hoja con dos hileras de los mismos dibujos, una de las cuales tiene dos intercambiados de lugar. El niño deberá identificar cuál de las dos series tiene los dibujos ordenados de la misma manera que la que vio antes. La prueba está compuesta por siete niveles de tres ensayos cada uno. En el primer nivel, los ensayos están formados por tres dibujos cada uno, y a medida que aumentan los niveles, aumenta en uno la cantidad de ítems, es decir, se presenta un dibujo más. La medida que se obtiene de SD es un puntaje final, logrado a partir de la suma de cada ensayo realizado correctamente multiplicado por el nivel en el que se encuentra dicho ensayo.

La prueba cuenta con un índice de fiabilidad aceptable (Alpha de Cronbach $=.77$ ) y con evidencias a favor de validez concurrente y discriminante (Injoque-Ricle, 2010).

Orden de Palabras (OP; Kaufman \& Kaufman, 1983). Es una de las pruebas que componen la Batería de Evaluación para niños de Kaufman (KABC, Kaufman Assessment Battery for Children, Kaufman \& Kaufman, 1983). Su objetivo es evaluar la MT. Se le presenta al niño una hoja en blanco, y se le lee una lista de palabras que corresponden a una serie de dibujos que aparecerán a continuación. Luego se le presenta una hoja con los dibujos correspondientes a esas palabras, alineados horizontalmente y el niño debe tocar los dibujos en el mismo orden en el que fueron mencionados. Se miden la cantidad de ensayos realizados correctamente por cada niño.

En el análisis psicométrico de la tarea se encontró un coeficiente Alpha de Cronbach de .66, lo cual indica una fiabilidad aceptable, y evidencias a favor de validez concurrente y discriminante (Injoque-Ricle, 2010).

Cubos (Wechsler, 1994). Es un subtest de Ejecución del Test de Inteligencia para niños de Wechsler-III (WISC-III; Wechsler, 1994). Tiene como objetivo evaluar la habilidad de analizar y sintetizar estímulos visuales abstractos. Cuenta con 12 ítems formados por diseños geométricos que el niño tiene que reproducir utilizando cubos rojos y blancos.

\section{Procedimiento}

Se trabajó con cada niño en forma individual en dos sesiones de aproximadamente 40 minutos cada una, separadas una de la otra por una semana, dentro de las instituciones educativas y durante el horario escolar, en un ambiente libre de ruidos. En la primer sesión se administró TOL, SD, OP y Cubos. En la segunda se administró la AWMA completa.

\section{Análisis de los datos}

Para el análisis de los ítems en primer lugar se calculó la dificultad de los mismos a través del porcentaje de respuestas correctas de cada uno, y en segundo lugar se estimó el Alpha de Cronbach para medir la fiabilidad por consistencia interna. Con respecto a la evidencia a favor de la validez concurrente y discriminante se realizaron correlaciones de Pearson.

\section{Resultados}

En cuanto al análisis de los ítems, se evaluó la dificultad, encontrando que la misma aumenta a medida que avanzan los niveles, a excepción del nivel 5 (ver Figura 1). Se obtuvo un coeficiente Alpha de Cronbach de .73, lo cual indica una fiabilidad aceptable. En cuanto a las evidencias a favor de la validez convergente y discriminante, para evaluar la primera se realizaron correlaciones con las pruebas de la AWMA, SD y OP, observando asociaciones positivas significativas en todos los casos (ver Tabla 2); y para evaluar la evidencia a favor de la validez discriminante se realizaron 
correlaciones con Cubos del WISC-III, no encontrando asociaciones significativas entre los puntajes de estas dos pruebas $(r=, 039 ; p=.577)$ (ver Tabla 2$)$.

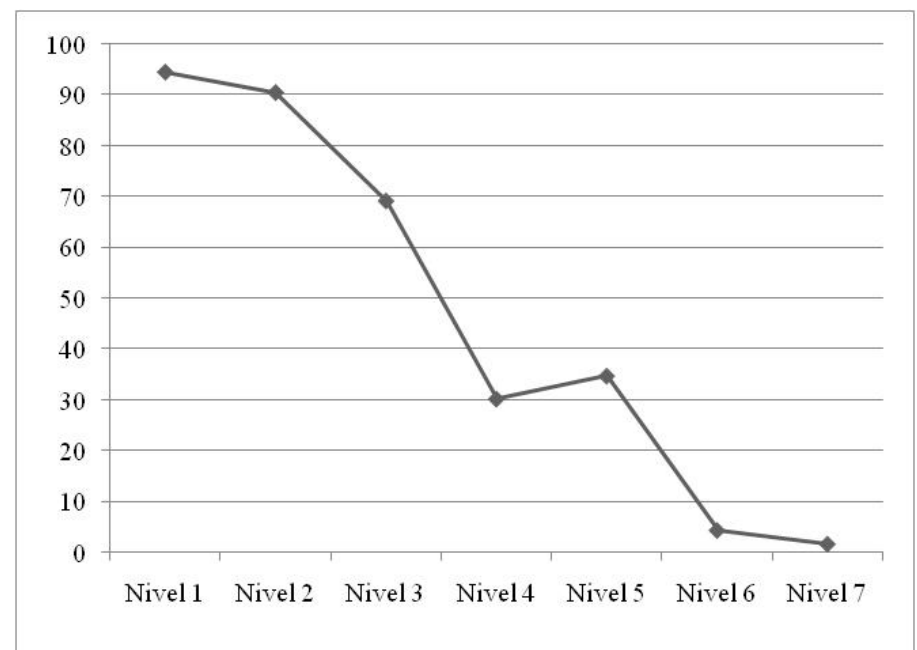

Figura 1. Porcentajes de aciertos en los distintos niveles de Torre de Londres.

Tabla 2

Correlaciones entre TOL, pruebas de la AWMA. SD. OP y Cubos

\begin{tabular}{lcc}
\hline \multirow{2}{*}{ Amplitud de dígitos } & \multicolumn{2}{c}{ TOL $^{\mathrm{a}}$} \\
\cline { 2 - 3 } Amplitud de palabras & .230 & $.001^{\mathrm{b} *}$ \\
Amplitud de no-palabras & .238 & $.001^{* *}$ \\
Matrices dinámicas & .287 & $.000^{* *}$ \\
Laberintos & .413 & $.000^{* *}$ \\
Amplitud viso-espacial & .383 & $.000^{* *}$ \\
Amplitud de oraciones & .422 & $.000^{* *}$ \\
Amplitud numérica & .456 & $.000^{* *}$ \\
Amplitud de dígitos inversos & .378 & $.000^{* *}$ \\
Figura diferente & .413 & $.000^{* *}$ \\
Señor X & .376 & $.000^{* *}$ \\
Recuerdo espacial & .414 & $.000^{* *}$ \\
Cubos & .339 & $.000^{* *}$ \\
\hline
\end{tabular}

Nota. $N$ = 210; a: Torre de Londres; b: r de Pearson; c:

Significación

$* * p<.01$

\section{Discusión}

El trabajo realizado permitió obtener una prueba válida y confiable, que permiten medir la planificación con cierto grado de confianza. Esto hace que el instrumento adaptado sea apto para ser utilizado con niños de 6, 8 y 11 años de clase media de la Ciudad Autónoma de Buenos Aires, Argentina.

\section{Estudio 2}

\section{Método}

\section{Participantes}

Se trabajó con 180 niños de ambos sexos (103 mujeres $-57.2 \%$ - y 77 varones), de clase media, distribuidos uniformemente en tres grupos de edad que concurrían una institución educativa pública de la Ciudad Autónoma de Buenos Aires, Argentina. Los grupos estuvieron compuestos por: 60 niños de 6 años, 60 de 8 años y 60 de 11 años (ver Tabla 3). Se siguió el mismo criterio de exclusión que en el Estudio 1, y tanto el acceso a las instituciones como la obtención de la autorización por parte de los padres tuvieron el mismo procedimiento.

Tabla 3

Distribución por sexo según grupo de edad del Estudio 2

\begin{tabular}{lcccccc}
\hline & \multicolumn{8}{c}{ Edad } \\
\cline { 2 - 7 } & \multicolumn{2}{c}{6} & \multicolumn{2}{c}{8} & \multicolumn{2}{c}{11} \\
& $\mathrm{~N}$ & $\%$ & $\mathrm{~N}$ & $\%$ & $\mathrm{~N}$ & $\%$ \\
\hline Mujeres & 25 & 41.7 & 35 & 58.3 & 43 & 71.7 \\
Varones & 35 & 58.3 & 25 & 41.7 & 17 & 28.3 \\
\hline Total & 60 & 100 & 60 & 100 & 60 & 100 \\
\hline
\end{tabular}

Instrumentos

Batería Automatizada de Memoria de Trabajo (Alloway, 2007; Injoque-Ricle, et al., 2011). A partir de los puntajes obtenidos en cada subtest de la AWMA, y siguiendo el modelo clásico de Baddeley y Hitch, se calcularon las tres puntuaciones compuestas: 1) Puntuación Compuesta del Bucle Fonológico, resultante de la sumatoria de los puntajes de Amplitud de Dígitos, Amplitud de Palabras y Amplitud de No-Palabras; 2) Puntuación Compuesta de la Agenda VisoEspacial, obtenida a través de la suma de los puntajes de Matrices Dinámicas, Amplitud Viso-Espacial y Laberintos; y 3) Puntuación Compuesta del Ejecutivo Central, que surge de la sumatoria de los puntajes de Amplitud de oraciones, Amplitud Numérica, Amplitud de Dígitos Inversos, Figura Diferente, Señor X y Recuerdo Espacial.

En relación a las propiedades psicométricas de la tarea, las puntuaciones compuestas cuentan con índices de fiabilidad altos (Puntuación Compuesta de la Agenda Viso-Espacial: Alpha de Cronbach $=.87$; Puntuación Compuesta del Ejecutivo Central: Alpha de Cronbach $=.91)$, $\mathrm{y}$ aceptables (Puntuación Compuesta del Bucle Fonológico: Alpha de Cronbach $=.65$ ). Se encontraron también evidencias a favor de la validez concurrente y discriminante (Injoque-Ricle, 2010)

Torre de Londres (Injoque-Ricle \& Burin, 2008b; Shallice, 1982). Se administró la versión definitiva validada en el Estudio 1.

\section{Procedimiento}

A cada uno de los 180 niños se les administraron los instrumentos de forma individual en la institución educativa, durante el horario escolar, en un ambiente libre de ruidos.

Análisis de los datos

Para evaluar las relaciones entre las Puntuaciones Compuestas de la AWMA y el puntaje de TOL en los 
distintos grupos y ver si éstas varían con la edad, como primer paso se analizó si existen diferencias en el rendimiento según la edad en la medida de planificación. Para ello se realizó un ANOVA de medidas independientes, seguido de un análisis post hoc, utilizando el contraste de Bonferroni. Con el fin de determinar si existen asociaciones entre las medidas de MT y la medida de planificación se realizaron correlaciones de Pearson en cada submuestra.

\section{Resultados}

En la Tabla 4 se presentan los estadísticos descriptivos de las Puntuaciones Compuestas y de TOL para cada grupo de edad.

Tabla 4.

Estadísticos descriptivos las Puntuaciones Compuestas de la AWMA y Torre de Londres para cada grupo de edad

PCBF $^{\mathrm{a}}$

PCAVE $^{\mathrm{b}}$

PCEC $^{\mathrm{C}}$

$\mathrm{TOL}^{\mathrm{d}}$

\begin{tabular}{lcccccccccccccccc} 
& $\mathrm{Med}^{\mathrm{e}}$ & $\mathrm{DE}^{\mathrm{f}}$ & $\mathrm{Min}^{\mathrm{g}}$ & $\mathrm{Max}^{\mathrm{h}}$ & $\mathrm{Med}^{\mathrm{e}}$ & $\mathrm{DE}^{\mathrm{f}}$ & $\mathrm{Min}^{\mathrm{g}}$ & $\mathrm{Max}^{\mathrm{h}}$ & $\mathrm{Med}^{\mathrm{e}}$ & $\mathrm{DE}^{\mathrm{f}}$ & $\operatorname{Min}^{\mathrm{g}}$ & $\mathrm{Max}^{\mathrm{h}}$ & $\mathrm{Med}^{\mathrm{e}}$ & $\mathrm{DE}^{\mathrm{f}}$ & $\operatorname{Min}^{\mathrm{g}}$ & $\mathrm{Max}^{\mathrm{h}}$ \\
\hline $6^{\mathrm{i}}$ & 14.27 & 2.121 & 9.00 & 19.00 & 13.56 & 4.22 & 4.67 & 23.67 & 7.63 & 2.56 & 2.33 & 14.50 & 18.70 & 9.69 & 5 & 48 \\
$8^{\mathrm{i}}$ & 16.69 & 2.42 & 11.00 & 20.67 & 20.24 & 3.72 & 13.00 & 29.00 & 11.09 & 2.75 & 4.83 & 18.17 & 24.32 & 10.45 & 10 & 50 \\
$11^{\mathrm{i}}$ & 19.11 & 3.01 & 11.67 & 25.67 & 25.32 & 3.37 & 18.67 & 33.00 & 15.73 & 3.14 & 8.50 & 25.17 & 29.27 & 11.98 & 6 & 56 \\
\hline
\end{tabular}

Nota. a: Puntuación Compuesta del Bucle Fonológico; b: Puntuación Compuesta de la Agenda Viso-Espacial; c: Puntuación Compuesta del Ejecutivo Central; d: Torre de Londres; e: Media; f: Desviación Estándar; g: Mínimo; h: Máximo; i: $n=60$

Tabla 5

Análisis Post hoc Torre de Londres

\begin{tabular}{lcc}
\hline & Dif Med $^{\mathrm{a}}$ & Sig. $^{\text {b }}$ \\
\hline $6-8$ & -5.62 & $.014^{*}$ \\
$6-11$ & -10.57 & $.000^{* *}$ \\
$8-11$ & -4.95 & $.038^{*}$ \\
\hline Nota. $n=60 ;$ a: Diferencia de Medias; b: Significación \\
$* p<.05 ;{ }^{* *} p<.01$
\end{tabular}

Tabla 6

Correlaciones entre Torre de Londres y las Puntuaciones Compuestas por grupo de edad

\begin{tabular}{lcccccc}
\hline & \multicolumn{6}{c}{ TOL $^{\mathrm{a}}$} \\
\cline { 2 - 8 } & \multicolumn{2}{c}{$6^{\mathrm{b}}$} & \multicolumn{3}{c}{$8^{\mathrm{b}}$} & \multicolumn{2}{c}{$11^{\mathrm{b}}$} \\
& $\mathrm{r}^{\mathrm{c}}$ & Sig. $^{\mathrm{d}}$ & $\mathrm{r}^{\mathrm{c}}$ & Sig. $^{\mathrm{d}}$ & $\mathrm{r}^{\mathrm{c}}$ & Sig. $^{\mathrm{d}}$ \\
\hline PCBF $^{\mathrm{e}}$ & .072 & .587 & .115 & .384 & .084 & .524 \\
PCAVE $^{\mathrm{f}}$ & .124 & .344 & .225 & .084 & .265 & $.041^{*}$ \\
PCEC $^{\mathrm{g}}$ & .106 & .422 & .272 & $.036^{*}$ & .343 & $.007^{* *}$ \\
\hline
\end{tabular}

Nota. a: Torre de Londres; b: $n=60$; c: r de Pearson; d: Significación; e: Puntuación Compuesta del Bucle Fonológico; f: Puntuación Compuesta de la Agenda VisoEspacial; g: Puntuación Compuesta del Ejecutivo Central $* p<.05 ; * * p<.01$

\section{Conclusiones y Discusión General}

Torre de Londres (Injoque-Ricle \& Burin, 2008b; Shallice, 1982) es una prueba usada como medida de planificación. Para la resolución de esta tarea, es necesario armar, mantener y ejecutar un plan de acción. Este plan implica múltiples etapas, que se van modificando a medida que se va ejecutando, y para poder llevarlo a cabo es necesaria la MT (Bull, et al., 2008; Gilhooly, Wynn, Phillips, Logie, \& Della Sala, 2002; Phillips, et al., 1999). En el proceso de validación realizado se constató la facilidad de la administración y un aumento de la dificultad de los ítems a medida que se avanza en los niveles. Se encontraron también niveles de fiabilidad aceptables y evidencias a favor de la validez concurrente y discriminante. Todo esto indica que la adaptación a nuestro medio socio-cultural es válida y confiable, y permite que las conclusiones arribadas en el presente estudio sean válidas para nuestro contexto.

El objetivo de este trabajo fue estudiar la relación de la MT y la planificación en niños de distintas edades y ver si esta relación varía con la edad. Para ello, en primer lugar se exploró si existía un rendimiento diferenciado en la medida de planificación en las distintas edades evaluadas, y luego, cuál era la relación entre la planificación y cada componente de la MT en cada subgrupo.

En cuanto al desarrollo evolutivo de la planificación, se observó que las puntuaciones obtenidas en TOL aumentan significativamente a medida que aumenta la edad, permitiendo concluir que existe un desarrollo diferenciado en la infancia, tal como hallaron Krikorian y cols. (1994b), y como se desprende del estudio de Lipina y cols. (2004).

Con respecto a la relación entre la MT y la resolución de TOL, del trabajo de Morris y cols. (1993) surge la pregunta de si se produce un repaso verbal activo durante la resolución de dicha prueba, ya que los hallazgos de estos autores se contraponen a las afirmaciones realizadas por una gran cantidad de investigadores, de que el componente verbal de la 
MT no tiene un rol en la resolución de esta tarea de planificación, y que solamente participan el componente visoespacial y el componente ejecutivo (e.g. Joyce \& Robins, 1991; Morice \& Delahunty, 1996; Owen, et al., 1996; Temple, et al., 1996; Welsh, et al., 1995). Los resultados del presente trabajo apoyan los hallazgos de Joyce y Robbins (1991), Morice y Delahunty (1996), Owen y cols. (1996), Temple y cols. (1996), y Welsh y cols. (1995), que sugieren que el BF no estaría implicado en la resolución de TOL, y sí la AVE y el EC, aunque según los resultados del presente estudio, no en todas las edades. Los datos indican que a los seis años no habría relación entre ninguno de los componentes de la MT y la planificación, lo que podría estar indicando que si bien existe una asociación entre la MT y la planificación, esta se da en niños más grandes, corroborando que la relación entre las medidas de MT y de planificación varía con la edad. A los ocho años, la relación sólo se observa con la medida del EC, mientras que a los once, la medida de planificación se asocia tanto con la medida del EC como con la medida de la AVE.

En base a los resultados surgen distintas hipótesis en cuanto a la relación en las diferentes edades entre TOL y los distintos componentes de la MT. Por un lado, el que a los ocho años no se haya encontrado una asociación significativa entre la medida de planificación y la de la AVE, y que de las asociaciones observadas a los once años la de mayor fuerza sea con el EC, podría estar indicando que si bien el componente viso-espacial de la MT está implicado en el desarrollo de esta tarea de planificación, es el componente ejecutivo de este sistema de memoria el que más peso tiene para su resolución. En cuanto a la falta de asociación con los tres componentes de la MT observada a los seis años, podría deberse a que a esa edad podrían ser otras funciones cognitivas y no la MT las que tengan una mayor implicancia en las habilidades necesarias para la resolución de TOL. Esto último requerirá futuros estudios en los que se evalúen en esa edad, además de la MT, diferentes funciones cognitivas relacionadas con las habilidades de planificación de tareas viso-espaciales, para poder determinar cuáles se asocian con dicha planificación y qué asociaciones tienen mayor fuerza, y por lo tanto, mayor implicancia en la resolución de esa tarea.

\section{Referencias bibliográficas}

Alloway, T. P. (2007). Automated Working Memory Assessment London: The Psychological Corporation.

Alloway, T. P., Gathercole, S. E., Willis, C., \& Adams, A. M. (2004). A structural analysis of working memory and related cognitive skills in young children. Journal of Experimental Child Psychology, 87, 85-106.

Anderson, V. A., Anderson, P., Northam, E., Jacobs, R., \& Mikiewicz, O. (2002). Relationship between cognitive and behavioral measures of executive function in children with brain disease. Child Neuropsychology, 8(4), 231-240.

Ashcraft, M. H. (1995). Cognitive psychology and simple arithmetic: A review and summary of new directions. Mathematical Cognition, 1, 3-34.

Baddeley, A. D. (1986). Working memory. Oxford: Clarendon Press.

Baddeley, A. D. (2000). The episodic buffer: a new component of working memory? Trends in Cognitive Sciences, 4(11), 417-423.

Baddeley, A. D. (2007). Working Memory, thought, and action. Oxford: Oxford University Press.

Revista Neuropsicologia Latinoamericana (2011), 3(2), 31-38
Baddeley, A. D., \& Hitch, G. J. (1974). Working memory. En G. H. Bower (Ed.), The psychology of learning and motivation: Advances in research and theory (Vol. 8, pp. 47-90). New York: Academic Press.

Baddeley, A. D., \& Logie, R. H. (1999). Working Memory: The multiple-component model. En A. Miyake \& P. Shah (Eds.), Models of Working Memory. Cambridge: Cambridge University Press.

Baddeley, A. D., Eysenck, M. W., \& Anderson, M. C. (2009). Memory. New York: Psychology Press.

Boghi, A., Rasetti, R., Avidano, F., Manzone, C., Orsi, L., D'Agata, F., et al. (2006). The effect of gender on planning: An fMRI study using the Tower of London task. Neuroimage, 33 (3), 999-1010.

Bull, R., Espy, K. A., \& Senn, T. E. (2004). A comparison of performance on the Towers of London and Hanoi in young children. Journal of Child Psychology and Psychiatry 45 (4), 743-754.

Bull, R., Espy, K. A., \& Wiebe, S. A. (2008). Short-Term Memory, Working Memory, and Executive Functioning in Preschoolers: Longitudinal Predictors of Mathematical Achievement at Age 7 Years. Developmental Neuropsychology, 33(3), 205-228.

Burin, D. I., Duarte, D. A., Prieto, G., \& Delgado, A. (2004). Memoria de trabajo viso-espacial y aptitud de Visualización. Cognitiva, 16, 95-113.

Cohen, G. (1996). Memory in the real world ( $2^{\circ}$ ed.). Hove: Psychology Press.

Fuster, J. M. (2002). Frontal lobe and cognitive development. Journal of Neurocytology, 31 (3-5), 373-385.

Gathercole, S. E., Alloway, T. P., Willis, C., \& Adams, A. (2006). Working memory in children with reading disabilities. Journal of Experimental Child Psychology 93, 265-281.

Gilhooly, K. J., Wynn, V., Phillips, L. H., Logie, R. H., \& Della Sala, S. (2002). Visuo-spatial and verbal working memory in the five-disc Tower of London task: An individual differences approach. Thinking and Reasoning, 8(3), 165178.

Hayes-Roth, B., \& Hayes-Roth, F. (1979). A cognitive model of planning. Cognitive Science, 3, 275-310.

Injoque-Ricle, I. (2010). Memoria de Trabajo: Evaluación de su desarrollo en niños. Facultad de Medicina, Universidad de Buenos Aires, Buenos Aires.

Injoque-Ricle, I., \& Burin, D. I. (2008a). Validación preliminar de una prueba de memoria de trabajo para niños: Serie de Dibujos. Memorias del I Congreso Internacional de Investigación y Práctica Profesional en Psicología, Tomo II, 331-332.

Injoque-Ricle, I., \& Burin, D. I. (2008b). Validez y fiabilidad de la prueba de Torre de Londres para niños: Un estudio preliminar. Revista Argentina de Neuropsicología, 11, 2131.

Injoque-Ricle, I., Calero, A., Alloway, T. P., \& Burin, D. I. (2011). Assessing Working Memory in Spanish-Speaking Children: Automated Working Memory Assessment Adaptation. Learning and Individual Differences, 21(1), 78-84.

Joyce, E. M., \& Robins, T. W. (1991). Frontal lobe function in Korsakoff and non-Korsakoff alcoholics: Planning and spatial working memory. Neuropsychologia, 29, 708-723.

Kaufman, A. S., \& Kaufman, N. L. (1983). Batería de evaluación de Kaufmann para niños. España: TEA.

Klahr, D. (1978). Goal formation, planning, and learning by preschool problem solvers or: "My socks are in the dryer". En R. Siegler (Ed.), Children's thinking: What develops? Hillside: Lawrence Erlbaum.

Krikorian, R., Bartok, J. A., \& Gay, N. (1994b). Tower of London procedure: A standard method and developmental data. 
Journal of Clinical and Experimental Neuropsychology, $16,840-850$.

Lezak, M. D. (1995). Neuropsychological assessment. New York: Oxford.

Lipina, S. J., Martelli, M. I., Vuelta, B. L., Injoque Ricle, I., \& Colombo, J. A. (2004). Pobreza y desempeño ejecutivo en alumnos preescolares de la Ciudad de Buenos Aires (República Argentina). Interdisciplinaria, 21(2), 153-193.

Luria, A. R. (1973). The working brain: An introduction to neuropsychology. New York: Basic Books.

Miyake, A., Friedman, N. P., Emerson, M. J., Witzki, A. H., Howerter, A., \& Wager, T. D. (2000). The unity and diversity of executive functions and their contributions to complex frontal lobe tasks: A latent variable analysis. Cognitive Psychology, 41, 49-100.

Morice, R., \& Delahunty, A. (1996). Frontal/executive impairments in schizophrenia. Schizophrenia Bulletin, 22, 125-137.

Morris, R. G., Ahmed, S., Syed, G. M., \& Toone, B. K. (1993). Neural correlates of planning ability: Frontal lobe activation during the Tower of London test. Neuropsychologia, 31, 1367-1378.

Neisser, U. (1967). Cognitive psychology. Nueva York: AppletonCentury-Crofts.

Newman, S. D., Carpenter, P. A., Varma, S., \& Just, M. A. (2003). Frontal and parietal participation in problem solving in the Tower of London: fMRI and computational modeling of planning and high-level perception. Neuropsychologia, 41(12), 1668-1682.

Owen, A. M., Downes, J. J., Sahakian, B. J., Polkey, C. E., \& Robbins, T. W. (1990). Planning and spatial working memory following frontal lobe lesions in man. Neuropsychologia, 28, 1021-1034.

Owen, A. M., Doyon, J., Petrides, M., \& Evans, A. C. (1996). Planning and spatial working memory: A positron emission tomography study in humans. European Journal of Neuroscience, 8, 353-364.

Phillips, L. H., Wynn, V., Gilhooly, K. J., Della Sala, S., \& Logie, R. H. (1999). The role of memory in the Tower of London task. Memory, 7(2), 209-231.

Shallice, T. (1982). Specific impairments of planning. Philosophical Transcripts of the Royal Society of London, 298(2), 199209.

Shallice, T. (1990). From neuropsychology to mental structure. New York: Cambridge University Press.

Simon, H. A. (1975). The functional equivalence of problem solving skills. Cognitive Psychology 7, 268-288.

Soprano, A. M. (2003). Evaluación de las funciones ejecutivas en el niño. Revista de Neurología, 37(1), 44-50.

Steinberg, L. (2007). Risk tasking in adolescence: New perspectives from brain and behavioral science. Current Directions in Psychological Science, 16, 55-59.

Stuss, D. T. (1992). Biological and physiological development of executive function. Brain and Cognition, 20, 8-23.

Stuss, D. T., \& Alexander, M. P. (2007). Is there a dysexecutive syndrome? Philosophical Transactions of the Royal Society of London, Series B, Biological Sciences, 362, 901915.

Swanson, H. L., \& Jerman, O. (2007). The influence of working memory on reading growth in subgroups of children with reading disabilities. Journal of Experimental Child Psychology, 96, 249-283.

Temple, C. M., Carney, R. A., \& Mullarkey, S. (1996). Frontal lobe function and executive skills in children with Turner's syndrome. Developmental Neuropsychology, 12, 343-363.

Walsh, K. H. (1978). Neuropsychology: A clinical approach. Edinburgh: Chirchill Livingstone.
Wechsler, D. (1994). Test de inteligencia para niños WISC-III, Manual. Buenos Aires: Paidós.

Welsh, M. C., Cicerello, A., Cuneo, K., \& Brennan, M. (1995). Error and temporal patterns in Tower of Hanoi performance: Cognitive mechanisms and individual differences. Journal of General Psychology, 122, 69-81. 